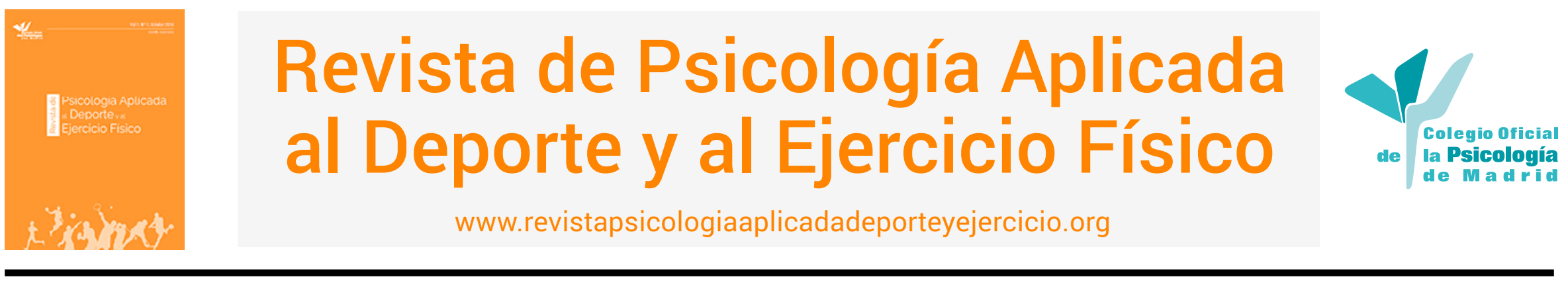

\title{
Desensibilización Sistemática para el control de la ansiedad: Un
} caso en atletismo

\author{
Rosendo Berengüí y María Á. Castejón \\ Universidad Católica de Murcia (UCAM), España
}

RESUMEN: El objetivo del trabajo fue aplicar la técnica de desensibilización sistemática a una atleta de velocidad de 19 años ( $\mathrm{N}=1$ ) que presentaba altos niveles de ansiedad competitiva. Se realizó una evaluación pre y post-intervención, mediante cuestionarios para valorar la ansiedad (SCAT) y sus características psicológicas relacionadas con en el deporte (IPED), así como mediante entrevista para valorar su vivencia del estrés y la ansiedad, y sus estrategias de afrontamiento. La desensibilización sistemática fue aplicada en nueve sesiones, pasando por cuatro fases: 1) entrenamiento en relajación, 2) construcción de la jerarquía de estímulos, 3) práctica en imaginación, y 4) entrenamiento en desensibilización sistemática. Los resultados indican una reducción de la ansiedad y un aumento de los recursos para hacer frente a la competición tras la intervención. Se concluye que la desensibilización sistemática es una técnica útil y eficaz para su empleo en el deporte.

PALABRAS CLAVES: desensibilización sistemática, ansiedad, estrés, activación, atletismo

\section{Systematic Desensitization for anxiety management: A case study in athletics}

ABSTRACT: The aim of the study was to apply the systematic desensitization technique to a 19-year-old female sprint athlete ( $\mathrm{N}=1$ ) who presented high levels of competitive anxiety. A pre- and post-intervention assessment was carried out, using questionnaires to assess anxiety (SCAT) and her psychological characteristics related to sport (IPED), as well as an interview to assess her experience of stress and anxiety, and her coping strategies. Systematic desensitization was applied in nine sessions, through four phases: 1) relaxation training, 2) stimulus hierarchy building, 3) imagery practice, and 4) systematic desensitization training. The results indicated a reduction in anxiety and an increase in resources to cope with competition after the intervention. It is concluded that systematic desensitization is a useful and effective technique for use in sport.

KEYWORDS: systematic desensitization, anxiety, stress, activation, athletics

\section{Dessensibilização sistemática para a gestão da ansiedade: um caso no atletismo}

RESUMO: O objectivo deste estudo foi aplicar a técnica de dessensibilização sistemática a uma atleta de velocidade de 19 anos ( $\mathrm{N}$ = 1) que apresentava altos níveis de ansiedade competitiva. Foi realizada uma avaliação pré e pós-intervenção utilizando questionários para avaliar a ansiedade (SCAT) e as características psicológicas relacionadas com o desporto (IPED), bem como uma entrevista para avaliar a sua experiência de stress e ansiedade, e as suas estratégias de coping. A dessensibilização sistemática foi aplicada em nove sessões, passando por quatro fases: 1) treino em relaxamento, 2) construção de hierarquia de estímulos, 3) prática imaginada, e 4) treino de dessensibilização sistemática. Os resultados indicaram uma redução da ansiedade e um aumento dos recursos para fazer face à concorrência após a intervenção. Conclui-se que a dessensibilização sistemática é uma técnica útil e eficaz para utilização no desporto.

PALAVRAS-CHAVE: dessensibilização sistemática, ansiedade, stress, activação, atletismo

Artículo recibido: 23/03/2021 | Artículo aceptado: 08/10/2021

Rosendo Berengüí es Dr. en Psicología en la Universidad Católica de Murcia (UCAM).

María Á. Castejón es Dra. en Psicología. Universidad Católica de Murcia (UCAM).

La correspondencia sobre este artículo debe enviarse a: Rosendo Berengüí Gil. al mail: rberengui@ucam.edu 
La activación del deportista y más concretamente el estrés y la ansiedad a nivel competitivo, han recibido una considerable atención en el campo de la Psicología del Deporte, siendo unos de los tópicos de estudio más destacados de la disciplina (Thomas et al., 2009).

En el deporte, el control del estrés, la ansiedad y los niveles de activación del deportista, resultan fundamentales para alcanzar un buen rendimiento. El nivel de activación óptimo es específico y diferente para cada deportista, y varía de acuerdo con la tarea a realizar y el deporte que se practique (Pérez-Cordoba et al., 2020). Además, en este ámbito la persona posee un antecedente considerable de carga física y psicológica, estando constantemente sometido a las más variadas influencias interpersonales y ambientales, y con independencia del sentido positivo o negativo que pueda tener un determinado estímulo, existen algunas situaciones características que contribuyen a convertir un estímulo en una situación estresante y generar ansiedad (Locatelli, 2018). Por ello, es necesario e imprescindible que los deportistas de todas las edades, y cualquiera que sea su nivel de habilidad, afronten efectivamente los factores estresantes, no solamente para alcanzar un elevado rendimiento, sino también para hacer del deporte una experiencia satisfactoria (Nicholls y Polman, 2007).

Existen diversos métodos y técnicas de intervención para el control del estado de activación del deportista, tanto con el objetivo de disminuirlo, como de incrementarlo (Cantú et al., 2018; Mellalieu et al., 2009; Rumbold et al., 2018; Thomas et al, 2009). Para tal propósito se han empleado, desde técnicas concretas de relajación, el entrenamiento atencional, establecimiento de objetivos, hipnosis, imaginería, rutinas de preactuación, pensamiento positivo, o el autodiálogo, entre otras muchas (Rumbold et al., 2012), hasta programas como el entrenamiento en inoculación del estrés (Meichenbaum, 1987) o el modelo COPE (Anshel y Gregory, 1990). Podemos considerar que los deportistas que cuentan con mayores recursos de afrontamiento, así como con un correcto entrenamiento de esas estrategias, tendrán mayor eficiencia en el control de las respuestas fisiológicas y emocionales causadas por el estrés, la ansiedad y las exigencias del entrenamiento y la competición (Cantú et al., 2018).

La revisión de Rumbold et al. (2012) sugiere que las intervenciones para el manejo del estrés con deportistas suelen ser generalmente efectivas, y las evidencias de diferentes intervenciones parecen indicar que, en su mayor parte, los componentes del estrés se optimizaron de una de las siguientes formas: a) se redujeron los estresores, b) se modificaron las valoraciones cognitivas, c) se redujeron los estados afectivos negativos y aumentaron los positivos y d) se facilitaron las conductas de afrontamiento eficaces.
En el presente estudio se realizó una intervención mediante el entrenamiento en Desensibilización Sistemática (DS). La técnica fue diseñada por Wolpe (1958) y se considera un procedimiento terapéutico que parte de la premisa de que la presencia de determinadas situaciones, estímulos o personas genera de forma automática respuestas de ansiedad. Partió del concepto de inhibición recíproca de Sherrington (1961), que planteaba que no pueden darse simultáneamente dos estados psicológicos incompatibles, de modo que la definición de lo que significa estar relajado excluye lo que representa estar ansioso. La técnica se basa en el contracondicionamiento progresivo que emplea la relajación como respuesta incompatible con la ansiedad. En este sentido, se contracondiciona una respuesta de ansiedad a un conjunto específico de estímulos, emparejando repetidamente representaciones imaginadas de situaciones que provocan ansiedad con la relajación, lo que da lugar a una disminución de la respuesta de ansiedad (Zemore, 1975). Finalmente se producirá la generalización, esto es, al asociar una respuesta concreta a una determinada situación, esa asociación se extenderá también a las distintas variaciones de esta situación (Wolpe, 1973).

La DS consta de cuatro pasos (Olivares et al., 2014): 1) entrenamiento en relajación, 2) construcción de la jerarquía, 3) evaluación y práctica en imaginación y 4) desensibilización sistemática propiamente dicha. En primer lugar, se debe instruir en relajación. Si bien Wolpe (1973) utilizó la relajación progresiva de Jacobson (1938), es posible emplear otras técnicas. A continuación, se construye la jerarquía de estímulos, elaborando una lista de estímulos o escenas con variaciones de ansiedad, desde situaciones de poca hasta máxima ansiedad. Tras la jerarquía, de debe evaluar y practicar la imaginación, y finalmente, aplicar la DS, combinando las respuestas de relajación frente a las de ansiedad.

La DS es una técnica clásica dentro de la psicología, que ha sido empleada con éxito en diversos ámbitos, como los trastornos fóbicos de diferentes tipos, trastornos de la alimentación, trastornos adictivos, trastornos somáticos, disfunciones sexuales, o asma, entre otros (Olivares et al., 2014; Vallejo-Slocker y Vallejo, 2016). De acuerdo con Vallejo-Slocker y Vallejo (2016), partiendo de su base teórica y en comparación con otras técnicas de exposición, la DS contribuye de mejor forma a la reducción de la ansiedad, a través de la relajación, la presentación gradual y el aprendizaje duradero de una respuesta alternativa bajo un contexto estimular variado que favorece la generalización. Según los autores, la DS también es una técnica bien recibida por los pacientes y además, un procedimiento flexible y con unas bases teóricas consolidadas. 
El objetivo de la intervención fue aplicar la técnica de desensibilización sistemática (Wolpe, 1958) a una atleta con altos niveles de ansiedad competitiva, basándonos en el procedimiento propuesto por Olivares et al. (2014).

\section{Método}

\section{Participantes}

La participante fue una mujer de 19 años, atleta especialista en pruebas de velocidad. Contaba con diez años de experiencia en su deporte, sobresaliendo por actuaciones y marcas desde categorías menores de competición. Entrenaba habitualmente cinco días a la semana, dependiendo del momento de la temporada, y una media de 14 horas de entrenamiento semanales. La deportista había registrado previamente buenos resultados a nivel nacional, si bien no los esperados por ella y su entrenador, de acuerdo a sus marcas personales.

La atleta había sido evaluada previamente y recibido asesoramiento a nivel de grupo (junto a otros/as compañeros/ as de club) por parte de quien realiza la intervención, si bien nunca había recibido apoyo o tratamiento psicológico de forma individual. El motivo de la intervención se debió al elevado estrés que le provoca la competición, cuya principal manifestación es una excesiva ansiedad competitiva, como demostró en la evaluación de grupo realizada.

La intervención fue realizada por un psicólogo con una experiencia de doce años en el área, Doctor en Psicología (Psicología Clínica y de la Salud) y titulaciones de Especialista Universitario y Máster en Psicología de la Actividad Física y el Deporte.

\section{Instrumentos}

Inventario Psicológico de Ejecución Deportiva (IPED). Se empleó la adaptación española (Hernández-Mendo, 2006), del Psychological Performance Inventory (PPI) de Loehr (1986). El IPED está compuesto de 42 ítems, divididos en siete subescalas: Autoconfianza, Control de Afrontamiento Negativo, Control Atencional, Control Visuo-Imaginativo, Nivel Motivacional, Control de Afrontamiento Positivo y Control Actitudinal. El rango de puntuaciones en todas las escalas oscila entre un mínimo de 6 y un máximo de 30. Tanto el estudio original (Hernández-Mendo, 2006), como el posterior estudio de replicación de sus propiedades psicométricas (Hernández-Mendo et al., 2014), afirmaron una fiabilidad y generalizabilidad satisfactorias del instrumento, confirmando el IPED como una herramienta adecuada, óptima y parsimoniosa. Diferentes estudios han encontrado una fiabilidad (alpha de Cronbach) entre $a=.64$ en Nivel Motivacional y $a=$ .81 en Control Visual e Imaginativo (Berengüí, 2011), y entre $a=.64$ en Control de Afrontamiento Negativo y $a=.79$ en Control Visual e Imaginativo (Raimundi et al., 2016).

Sport Competition Anxiety Test (SCAT). EI SCAT (Martens et al., 1990) está formado por 15 ítems y constituye una medida específica de ansiedad rasgo en el contexto deportivo, que evalúa la tendencia individual a percibir las situaciones competitivas como amenazantes y para responder a esas situaciones con sentimientos de aprensión y tensión. En el estudio de Berengüí (2011) con deportistas españoles se obtuvo una fiabilidad (alpha de Cronbach) $a=.85$.

Competitive State Anxiety Inventory (CSAI-2). Para evaluar la ansiedad estado previa a la competición, se empleó la adaptación de Tabernero y Márquez (1994) del CSAI-2, original de Martens et al. (1990). El cuestionario incluye 27 ítems que se dividen en tres subescalas: Ansiedad cognitiva, Ansiedad somática y Autoconfianza. Respecto a su fiabilidad, Arruza et al. (2012) obtienen $a=.91$ en Ansiedad cognitiva, $a=.93$ en Ansiedad somática, y $a=.90$ en Autoconfianza.

Entrevista. Se confeccionó una entrevista semiestructurada con el objetivo de recabar mayor información sobre todos los aspectos relevantes relacionados con el caso de la deportista, adaptando el entrevistador la secuencia y presentación de las preguntas de acuerdo a las características de la entrevistada (Hernández-Mendo et al., 2018). Se plantearon preguntas abiertas que abordaron el modo en que la atleta vivía su deporte y la importancia que le concedía en su vida cotidiana, el apoyo social real y percibido del que disponía, y su grado de control o habilidad respecto a diferentes variables relacionadas con el rendimiento deportivo (autoconfianza, motivación, concentración, visualización). La parte principal de la entrevista estuvo orientada a indagar en el origen o causas de su ansiedad, el control de la activación que realizaba en competición y los recursos de afrontamiento empleados.

\section{Procedimiento}

En primer lugar, previo a la intervención con DS, se realizó la evaluación en dos momentos, primero con la aplicación de los cuestionarios tras una sesión de entrenamiento de la deportista y posteriormente se realizó la entrevista.

La intervención mediante DS (Wolpe, 1958) se llevó a cabo durante nueve sesiones de aproximadamente una hora de duración cada sesión. 


\section{Entrenamiento en relajación}

En la primera sesión se realizó una sesión informativa en la que se expusieron los resultados obtenidos y el plan de trabajo a seguir. A continuación, se aportó información educacional sobre la importancia de una adecuada respiración, y sus beneficios asociados. Tras comprobar el predominio de la respiración torácica, se realizaron ejercicios para el trabajo en respiración abdominal. Finalizó la sesión con el recordatorio de la necesidad de trabajar en casa y en los entrenamientos el proceso de respiración adecuado.

En la segunda sesión se continuó instruyendo sobre la correcta respiración. A continuación, se inició el entrenamiento en relajación. Entre los posibles tipos, se optó por la relajación condicionada propuesta por Paul (1966), ya que la atleta afirmó que esa técnica le gustaba y le era fácil de emplear. Este tipo de relajación se basa en que la deportista asocie la relajación conseguida mediante la respiración a palabras o frases que se debe repetir a sí misma. Por ejemplo, entre otras empleó "estoy tranquila", "paz" o "estoy muy relajada".

\section{Construcción de la jerarquía}

En la tercera sesión se planteó el uso de la desensibilización sistemática como técnica que podría tener un resultado positivo, y se ofreció información sobre la técnica y su forma de proceder.

A continuación, se indagó sobre las situaciones que más ansiedad le provocaban a la hora de afrontar la competición. La atleta propuso una serie de situaciones que le inducían alta activación, concretamente las siguientes: salida a la pista para competir; charla con su entrenador, con instrucciones técnicas y tácticas; situada en los tacos de salida; calentamiento previo; salidas de calentamiento; en cama, la noche previa a la competición; preparando la equipación necesaria para competir; en el coche, en el desplazamiento a la competición; llegada a la instalación; ver a las rivales conocidas a las que se va a enfrentar; desayuno o comida antes de competir. Cada una de esas escenas fueron anotadas en una tarjeta.

El paso siguiente consistió en ordenar las tarjetas y graduar esas situaciones ansiógenas, elaborando la jerarquía según el grado de ansiedad que le provocaban, de menor a mayor intensidad. Tras su análisis, la lista quedó como sigue a continuación:

1. Desayuno o comida previos a la competición

2. Preparando la equipación

3. Desplazamiento en coche a la competición

4. Llegada a la instalación
5. Ver a las rivales

6. En cama, la noche previa a la competición

7. Charla previa con el entrenador

8. Calentamiento

9. Salida a la pista para competir

10. Salidas de calentamiento

11. En tacos de salida

\section{Práctica en imaginación}

En la cuarta sesión se realizó la evaluación de la capacidad de visualización de la deportista. Se le pidió, por ejemplo, que se visualizara a ella misma trotando en su pista habitual de entrenamiento, que realizara salidas de tacos, que intentara verse tanto desde su propia perspectiva como desde fuera, y que visualizara a velocidad real y a "cámara lenta". Acorde a la puntuación obtenida en el IPED, la atleta mostró una adecuada habilidad para imaginar cuestiones y circunstancias relacionadas con su deporte, con apropiado control de las imágenes y viveza de las mismas. En la sesión se introduce una visualización inicial de situaciones ansiógenas. Concretamente, se pedía la visualización de la última competición realizada. La atleta manifestó que el escenario le generaba nerviosismo. Tras volver a trabajar la relajación, la sesión terminó con el recordatorio de la necesidad de realizar lo practicado tanto en casa como en los entrenamientos.

\section{Entrenamiento en DS}

Las siguientes sesiones, hasta el final de la intervención, se comenzaron las sesiones con ejercicios de visualización y relajación. Posteriormente se pasaba a trabajar las situaciones planteadas en la jerarquía de situaciones generadoras de ansiedad. Se pidió a la deportista que imaginara la situación concreta, que la sintiera en primera persona, desde su propia perspectiva. En cada escena, la deportista debía levantar la mano para indicar que no se sentía emocionalmente bien con la imagen, y en esos casos se le pedía que se abandonara la situación y se centrara en la relajación. Después se le sugería que volviera a la escena previa que ya había superado, para posteriormente volver de nuevo a la escena no superada. Cuando una situación ya no generaba malestar, la atleta lo manifestaba verbalmente, se repetía de nuevo, y cuando ya no producía ansiedad en dos ocasiones consecutivas, se avanzaba a la siguiente.

En las dos primeras situaciones, las que graduó como de menor intensidad, la atleta afırmó que le costaba imaginar bien las situaciones debido a la incomodidad que le provoca- 
Tabla 1. Resumen del contenido de las sesiones de DS

\begin{tabular}{ll}
\hline Sesión & Contenido \\
\hline Primera & $\begin{array}{l}\text { Exposición de resultados de evaluación. } \\
\text { Educación en respiración. Ejercicios de respiración abdominal. } \\
\text { Técnicas de relajación. } \\
\text { Recordatorio de trabajo en casa y en entrenamientos. }\end{array}$ \\
\hline Segunda & Respiración. Entrenamiento en relajación condicionada. \\
\hline Tercera & Recordatorio de trabajo en casa y en entrenamientos. \\
\hline Cuarta & Construcción de la jerarquía: situaciones generadoras de ansiedad y graduación de mayor a menor. \\
\hline Quinta a octava & Evaluación de la capacidad de visualización. \\
& Visualización inicial de situaciones ansiógenas. \\
\hline Novena & Jerarquía de situaciones generadoras de ansiedad. \\
\hline
\end{tabular}

ban. Con el paso de las sesiones fue avanzando en control y viveza de las imágenes y fue superando cada situación. En las sesiones, la parte dedicada a la jerarquía se comenzaba con un repaso a las situaciones superadas previamente.

Se dedicó un tiempo variable hasta superar cada una de las once escenas, siendo las tres últimas las que requirieron de más tiempo. En la última sesión se realizó un repaso final a todas las situaciones, manifestando la atleta que no sentía ansiedad con esas imágenes.

La evaluación final fue realizada doce días después de la última sesión.

En todo momento la deportista fue informada de los objetivos y fases de la intervención, y de la privacidad y la confidencialidad de los datos aportados, siguiendo la Declaración de Helsinki (World Medical Association, 2013).

\section{Resultados}

\section{Evaluación inicial}

Para la evaluación de la intervención se emplearon el Sport Competition Anxiety Test (SCAT) y el Inventario Psicológico de Ejecución Deportiva (IPED). Además, se aplicó el CSAI-2 para evaluar su ansiedad estado en competición.

Partiendo de los datos de los cuestionarios, en la evaluación previa a la intervención, la deportista obtuvo en el Sport Competition Anxiety Test (SCAT) una puntuación de 28, por lo que podemos considerar que manifestaba un nivel alto de ansiedad competitiva. Además, se disponía de datos previos de la deportista antes de la intervención, que sirvieron de ayuda para analizar su ansiedad, concretamente, las puntuaciones en el Sixteen Personality Factor Questionnaire,

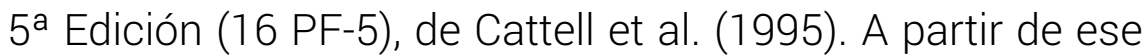
cuestionario, se comprobó que la atleta presentaba una alta ansiedad, teniendo en cuenta que se situaba en un decatipo de 8 en el factor Ansiedad y además, en las escalas primarias que contribuyen a esa dimensión sus decatipos eran 5 en Estabilidad Emocional, 6 en Vigilancia, 7 en Aprensión y 8 en Tensión.

En el Inventario Psicológico de Ejecución Deportiva (IPED) registró las siguientes puntuaciones: Autoconfian$z a=26$, Control de afrontamiento negativo $=15$, Control de la atención= 17, Capacidad de visualización e imaginativa $=25$, Nivel motivacional $=28$, Control de afrontamiento positivo $=$ 24 , Control actitudinal $=24$. A partir de esos datos, a nivel cualitativo podemos destacar que la atleta presentaba altos niveles de autoconfianza y nivel motivacional, y aceptables en cuanto a capacidad visuo-imaginativa, control de afrontamiento positivo y actitudinal, Sin embargo, destacaron sus bajos niveles de control de afrontamiento negativo y control de la atención.

En cuanto a la entrevista, la deportista aportó muchos datos relevantes sobre su experiencia en el deporte y en la competición. Manifestaba que disfrutaba mucho de su deporte ("es algo que me gusta y que se me da bien"; "es algo muy importante en mi vida"), y de los entrenamientos ("comparto mucho tiempo con amigas y amigos"; "mis mejores amigos practican también este deporte"; "entrenar es una rutina más de mi día a día... no sabría qué hacer si no entrenara"). Sus objetivos para la temporada eran conseguir una medalla en el 
campeonato nacional de su categoría y batir su marca personal.

Respecto a la competición, afirmó que siempre acudía a competir con confianza y actitud positiva, si bien una vez allí no podía conseguir una concentración óptima. Consideraba que su rendimiento real en competición no era el esperado, tanto por ella como por su entrenador ("siempre llego muy bien a los campeonatos, pero nunca es mi día"). Atribuyó ese rendimiento no deseado a sus "nervios al competir", a no saber manejar los momentos estresantes y a la alta ansiedad que registraba en competición, que se manifestaba tanto a nivel cognitivo ("tengo miedo y no consigo quitarme los malos pensamientos"; "es imposible centrarme en la carrera"; "la cabeza me da muchas vueltas"), como a nivel somático ("me sudan muchísimo las manos"; "parece que el corazón me vaya a reventar"; "mis músculos se endurecen como una piedra"). Además, esa alta ansiedad le impedía alcanzar una adecuada concentración, admitiendo que en las situaciones de estrés y en los momentos de alta ansiedad no disponía de recursos de afrontamiento para hacerles frente, y no empleaba por tanto ningún método o técnica para controlar su excesiva activación física y psicológica.

Consideraba que en su vida cotidiana también era usual manifestar ansiedad en diferentes situaciones, si bien no de forma tan alta y desproporcionada como en el deporte. Afirmaba también que en ningún momento se había sentido presionada por su entrenador o sus padres, más bien al contrario, siempre tuvo la percepción de que se encontraba muy respaldada y apoyada por ellos, tanto en los buenos como en los malos momentos, aunque admitió que temía hacerlo mal en competición por ellos ("no quiero defraudarlos").

Entre las sesiones de entrenamiento la atleta compitió en dos ocasiones, a nivel regional. En una admitió que su rendimiento fue bueno, pues estuvo cerca de su marca personal, y en la otra, el resultado fue estimado como pobre, pues relataba que era un día con mucho viento que impedía correr adecuadamente, y que incluso el viento invalidó la marca conseguida. Se aplicó el CSAI-2 para comprobar sus puntuaciones en ansiedad estado. En la primera competición la deportista registró puntuaciones de 27 en Ansiedad cognitiva, 26 Ansiedad somática y 27 en Autoconfianza. En la segunda, competición la deportista registró puntuaciones de 25 en Ansiedad cognitiva, 26 Ansiedad somática y 25 en Autoconfianza.

\section{Evaluación final}

Seis días después de la última sesión la atleta acudió a competir al campeonato nacional de su categoría. La depor- tista obtuvo la medalla de oro en su prueba y realizó su mejor marca personal. La semana siguiente a la competición se realizó la evaluación final, que consistió en la aplicación de dos cuestionarios y una entrevista.

En los cuestionarios de evaluación, la deportista registró en el Sport Competition Anxiety Test (SCAT) una puntuación de 22, bajando su nivel de ansiedad competitiva respecto a la evaluación inicial. El Inventario Psicológico de Ejecución Deportiva (IPED) las puntuaciones fueron las siguientes: Autoconfianza $=28$, Control de afrontamiento negativo $=22$, Control de la atención= 23, Capacidad de visualización e imaginativa $=26$, Nivel motivacional $=29$, Control de afrontamiento positivo $=25$, Control actitudinal $=26$. Como se puede comprobar, la atleta obtuvo mejores puntuaciones tras la intervención en varias escalas, si bien destacan las puntuaciones en las escalas de Control de Afrontamiento Negativo y Control de la Atención, en las que su nivel de control aumentó considerablemente.

Además, mediante entrevista se corroboraron esas mejoras. La atleta afirmaba que no sintió miedo a competir, y si bien sentía cierto grado de ansiedad, ese nivel no era tan alto ni incapacitante como en otras competiciones ("claro... un campeonato es siempre un campeonato... había nervios, pero no fue igual"). También, aunque se produjeron ciertas reacciones somáticas y cognitivas como en ocasiones anteriores, éstas no tuvieron la misma intensidad, y pudo mantenerse concentrada durante los dos días de competición ("me fueron muy útiles los ejercicios de respiración para relajarme... usé la respiración en todo momento, incluso la noche antes de la final, me ayudó mucho para poder dormir bien"). Del mismo modo, planteaba que, tras el resultado obtenido, su confianza y motivación eran muy altas.

Tabla 2. Puntuaciones pre y post-intervención en los cuestionarios de evaluación

\begin{tabular}{lccc}
\hline Escala & $\begin{array}{c}\text { Pre- } \\
\text { intervención }\end{array}$ & $\begin{array}{c}\text { Post- } \\
\text { intervención }\end{array}$ & Diferencia \\
\hline SCAT & 28 & 22 & -6 \\
\hline Autoconfianza & 26 & 28 & +2 \\
\hline $\begin{array}{l}\text { Control de Afrontamiento } \\
\text { Negativo }\end{array}$ & 15 & 22 & +7 \\
\hline Control Atencional & 17 & 23 & +6 \\
\hline Control Visuo-Imaginativo & 25 & 26 & +1 \\
\hline Nivel Motivacional & 28 & 29 & +1 \\
\hline $\begin{array}{l}\text { Control de Afrontamiento } \\
\text { Positivo }\end{array}$ & 24 & 25 & +1 \\
\hline Control Actitudinal & 24 & 26 & +2 \\
\hline
\end{tabular}




\section{Discusión}

El objetivo de la intervención fue aplicar la técnica de desensibilización sistemática (Wolpe, 1958) a una atleta con altos niveles de ansiedad en competición. Los resultados fueron positivos, pues la atleta consiguió mantener su ansiedad en un nivel moderado o aceptable en competición, el cuál no perjudicó su rendimiento, y le permitió actuar en un estado óptimo a nivel físico y psicológico. Además, la atleta alcanzó los objetivos personales y deportivos fijados para la temporada.

Los datos obtenidos tras la evaluación final mostraron mejoras en Control de Afrontamiento Negativo y la disminución en la puntuación del Sport Competition Anxiety Test. A partir de ese mejor control de afrontamiento podemos advertir que la atleta logró un mejor dominio cognitivo y conductual a la hora de enfrentarse a situaciones deportivas adversas (Hernández-Mendo, 2006). Asimismo, la puntuación de ansiedad en el SCAT mostró una menor tendencia a percibir las situaciones competitivas como amenazantes y para responder de menor forma a esas situaciones con sentimientos de aprensión y tensión (Martens et al., 1990). Respecto a la escala Control de la Atención, la atleta alcanzó un mejor control de la concentración y capacidad para atender a la gran variedad de estímulos y situaciones que se presentan, manteniendo un estado de alerta, focalizado en los eventos destacados e ignorando los irrelevantes (Campos-Salinas et al., 2019). Las mejoras en ansiedad y atención fueron también confirmadas mediante entrevista, afırmando la deportista que, aunque sintió ciertas manifestaciones cognitivas y somáticas, se mantuvo calmada y concentrada, sin gastar excesiva energía como en competiciones anteriores. Tampoco tuvo miedo ni aparecieron pensamientos negativos en las diferentes situaciones que tuvo que afrontar.

Debemos tener en cuenta la evaluación previa de personalidad de la deportista, con puntuaciones altas en el factor Ansiedad, que definen a personas reactivas, suspicaces, aprensivas y tensas (Russell y Karol, 2005), con tendencia a sentirse presionados, irritables, sobreexcitados y preocupados (Karson et al., 2003). Partiendo de una concepción disposicional de la personalidad, asumiendo que es responsable de los patrones característicos de pensamiento, emoción y comportamiento del individuo, y que permanecen relativamente invariables a lo largo del tiempo y las situaciones (Berengüí y López-Walle, 2018), podemos comprender que la disposición ansiosa de la atleta hará que sus niveles de ansiedad habitualmente sean moderados a altos. Sin embargo, recursos de afrontamiento como los aportados y entrenados sirven para afrontar el estrés de forma adecuada y manejar de forma adaptativa la ansiedad y las demandas de la competición.
También un dato destacado es que, a pesar de sus niveles de ansiedad, la autoconfianza de la atleta no fue un aspecto que se viera perjudicado, como muestra sus puntuaciones en el IPED, ni que disminuyera en competición, como generalmente han mostrado los estudios que han empleado el CSAI-2 (Martens et al., 1990). De hecho, se ha llegado a plantear que la autoconfianza puede considerarse un factor de resiliencia que puede proteger contra los efectos debilitantes de la ansiedad (Mellalieu et al., 2009), y es por ello que la confianza de la deportista podría considerarse en este caso un factor esencial que ha podido ayudar y contribuir en el proceso seguido.

Como conclusión, y a partir de los resultados obtenidos, podemos señalar que en este contexto la DS se muestra como una técnica útil y eficaz para el trabajo sobre la ansiedad en el deporte. La DS ha sido mejor acogida que otras técnicas de exposición para la extinción de fobias condicionadas y ansiedades inadaptadas (Labrador y Crespo, 2008), y para Vallejo-Slocker y Vallejo (2016) ello se puede deber a que la DS se asienta sobre principios sólidos que en ocasiones pueden haber favorecido su elección frente a otras terapias, y también que, desde un punto de vista práctico, el procedimiento de aplicación de la DS puede provocar menos reactividad en los pacientes y favorecer la adherencia al tratamiento. Sin embargo, en el deporte siguen siendo escasos las experiencias de aplicación de la DS (e.g., Cantón et al., 2009; Cecil et al., 2009; Tabatabai y Badami, 2019).

Debemos tener en cuenta la principal limitación del estudio, que es esencialmente su propia naturaleza, pues al tratarse de un estudio de caso único no es posible generalizar los resultados, y por ello sería conveniente futuros estudios que apliquen la técnica en otros casos, y repliquen los efectos en un mayor número de sujetos. También sería apropiado evaluar las intervenciones con diferentes instrumentos a los empleados en este estudio.

\section{Aplicaciones prácticas}

Partiendo del perjudicial impacto que unos inadecuados niveles de estrés y ansiedad pueden tener sobre el rendimiento y la salud del deportista (Locatelli, 2018; Nicholls y Polman, 2007), son necesarias intervenciones que doten a los deportistas de adecuados recursos de afrontamiento, que impacten positivamente sobre su activación, y fomenten finalmente una experiencia deportiva positiva y su bienestar general.

A pesar de la limitación del presente estudio, creemos que los resultados positivos que se desprenden de esta intervención pueden animar al uso de la DS, como otra técni- 
ca útil y fiable para el tratamiento del estrés y la ansiedad competitiva. Además, el procedimiento seguido aquí puede servir de apoyo o guía para la aplicación de la DS en deportistas de diferentes modalidades.

Además de la DS, y numerosas técnicas provenientes de la psicología clínica, pueden emplearse diversas técnicas y procedimientos para el manejo del estrés y la ansiedad. Técnicas de respiración y relajación, el biofeedback, el entrenamiento atencional, el establecimiento de objetivos, la hipnosis, la imaginería, rutinas de preactuación, el pensamiento positivo, o el autodiálogo, entre otras muchas (Hanton et al., 2009; Rumbold et al., 2012, 2018), se han mostrado como procedimientos válidos y con alta efectividad para el control de los niveles de excesiva activación del deportista.

Además debemos tener presentes las evidencias que confirman su utilidad, pues los programas de intervención han demostrado resultados positivos y significativos, demostrándose que hasta el $70 \%$ de intervenciones que han empleado técnicas de relajación han reducido la ansiedad, y un 65\% los programas basados en imágenes (Rumbold et al., 2012), y además se obtienen buenos resultados para reducir la ansiedad cognitiva y somática cuando se emplea el auto-habla dentro de programas cognitivos y multimodales (Georgakaki y Karakasidou, 2017; Rumbold et al., 2012). También, un meta-análisis reciente (Ong y Chua, 2021) concluye que las intervenciones psicológicas sobre la ansiedad competitiva muestran que sus efectos parecen ser mayores para los atletas de niveles más altos de competición, en comparación con los de niveles más bajos, y que se obtienen tamaños del efecto de medios a elevados para la ansiedad cognitiva y la autoconfianza, siendo en ansiedad somática de pequeña a mediana la intensidad.

Finalmente, coincidimos con Olmedilla-Caballero et al. (2020) en la convicción de que los estudios de caso son necesarios para divulgar la parte aplicada de la Psicología del Deporte, y así poder ofrecer a los profesionales de esta área herramientas útiles para su trabajo, y es por ello que consideramos que se precisa de más estudios que propongan intervenciones que empleen diferentes técnicas de intervención, en deportistas de variadas disciplinas.

\section{Referencias}

Anshel, M. A. y Gregory, W. L. (1990). The effectiveness of a stress training program in coping with criticism in sport: A test of the COPE model. Journal of Sport Behavior, 13(4), 194-217.

Arruza, J. A., González, O., Palacios, M., Arribas, S. y Cecchini, J.A. (2012). Validación del Competitive State Anxiety Inventory 2 Reducido (CSAI-2 RE) mediante una aplicación web. Revista
Internacional de Medicina y Ciencias de la Actividad Física y del Deporte, 12(47), 539-556.

Berengüí, R. (2011). Personalidad, características psicológicas y lesiones en el deporte de competición: Análisis de relaciones e incidencia en jóvenes deportistas [Tesis doctoral no publicada]. Universidad de Murcia

Berengüí, R. y López-Walle, J. M. (2018). Personalidad y deporte. En R. Berengüi y J. M. López-Walle (Eds), Introducción a la Psicología del Deporte (pp. 65-98). EOS Editorial.

Campos-Salinas, J. A., Berengüí, R. y Hernández-Mendo, A. (2019). Inventario Psicológico de Ejecución Deportiva para Árbitros (IPEDA): Adaptación y Propiedades de Medida. Revista Iberoamericana de Diagnóstico y Evaluación - e Avaliação Psicológica, 53(4), 141-152. https://doi.org/10.21865/RIDEP53.4.11

Cantón, E., Checa, I. y Ortín, F. J. (2009). Intervención psicológica con un nadador de medio acuático abierto. Cuadernos de Psicología del Deporte, 9(2), 57-65.

Cantú, A., Reynoso, L. F. y Aragón, P. O. (2018). Técnicas de intervención en Psicología del Deporte. En R. Berengüi y J. M. López-Walle (Eds), Introducción a la Psicología del Deporte (pp. 385-426). EOS Editorial.

Cattell, R. B., Cattell, A. K. S. y Cattell, H. E. P (1995). 16PF5. Adaptación Española. TEA Ediciones.

Cecil, S., Brandon, R. y Moore J. (2009). An integrated multi-disciplinary support service for an injured rugby union lock. En B. Hemmings y T. Holder (Eds.), Applied Sport Psychology: A Case-Based Approach (pp. 203-221). Wiley-Blackwell.

Georgakaki, S. K. y Karakasidou, E. (2017). The Effects of Motivational Self-Talk on Competitive Anxiety and Self-Compassion: A Brief Training Program among Competitive Swimmers. Psychology, 8(5), 677-699. https://doi.org/10.4236/psych.2017.85044

Hanton, S., Thomas, O. y Mellalieu, S. D. (2009). Management of competitive stress in elite sport. En B. W. Brewer (Ed.), Sport Psychology (pp. 30-42). Wiley-Blackwell.

Hernández-Mendo, A. (2006). Un cuestionario para la evaluación psicológica de la ejecución deportiva: Estudio complementario entre TCT y TRI. Revista de Psicología del Deporte, 15(1), 71-93.

Hernández-Mendo, A., Morales-Sánchez, V. y Peñalver, I. (2014). Replicación de las propiedades psicométricas del Inventario Psicológico de Ejecución Deportiva. Revista de Psicología del Deporte, 23(2), 311-324.

Hernández-Mendo, A., Morales, V., Reigal, R., Juárez, R. y Ortigosa, J. M. (2018). Evaluación psicosocial en el deporte. En R. Berengüi y J. M. López-Walle (Eds), Introducción a la Psicología del Deporte (pp. 345-383). EOS Editorial.

Jacobson, E. (1938). Progressive relaxation. University of Chicago Press.

Karson, M., Karson, S. y O’Dell, J. (2003). 16 PF-5. Una guía para su interpretación en la práctica clínica. TEA Ediciones.

Labrador, F. J. y Crespo M. (2008). Desensibilización sistemática. En F. J. Labrador (Coord.), Técnicas de modificación de conducta (pp. 243-259). Pirámide.

Locatelli, L. (2018). Activación del deportista. En R. Berengüi y J. M. López-Walle (Eds), Introducción a la Psicología del Deporte (pp. 99-121). EOS Editorial.

Loehr, J. E. (1986). Mental toughness training for sports: Achieving athletic excellence. Stephen Greene Press. 
Martens, R., Vealey, R. y Burton, D. (1990). Competitive anxiety in Sport. Human Kinetics.

Meichenbaum, D. (1987). Manual de Inoculación de Estrés. Martínez Roca.

Mellalieu, S. D., Hanton, S. y Fletcher, D. (2009). A competitive anxiety review: Recent directions in sport psychology research. Nova Science Publishers.

Nicholls, A. R. y Polman, R. C. (2007). Coping in sport: A systematic review. Journal of Sports Sciences, 25(1), 11-31. https://doi. org/10.1080/02640410600630654

Olivares, J., Méndez, F. X. y Beléndez, M. (2014). La desensibilización sistemática. En J. Olivares y F. X. Méndez (Eds.), Técnicas de modificación de conducta (pp. 79-112). Biblioteca Nueva.

Olmedilla-Caballero, B., Moreno-Fernández, I. M., Gómez-Espejo, V. y Olmedilla-Zafra, A. (2020). Preparación psicológica para los Juegos Paralímpicos y afrontamiento de lesión: un caso en taekwondo. Revista de Psicología Aplicada al Deporte y al Ejercicio Físico, 5(1), Artículo e2, 1-13. https://doi.org/10.5093/rpadef2020a4

Ong, N. C. y Chua, J. H. (2021). Effects of psychological interventions on competitive anxiety in sport: A meta-analysis. Psychology of Sport \& Exercise, 52, 101836. https://doi.org/10.1016/j. psychsport.2020.101836

Paul, G. L. (1966). Insight vs. desensitization in psychotherapy: An experiment in anxiety reduction. Standford University Press.

Pérez-Córdoba, E. A., Estrada, O., Gutiérrez, M. T. y Ramírez, O. (2020). Nivel de activación óptimo y rendimiento en un jugador de fútbol no profesional. Revista de Psicología Aplicada al Deporte y al Ejercicio Físico, 5(1), Artículo e5. https://doi.org/10.5093/ rpadef2020a7

Raimundi, M. J., Reigal, R. E. y Hernández-Mendo, A. (2016). Adaptación argentina del Inventario Psicológico de Ejecución Deportiva (IPED): validez, fiabilidad y precisión. Cuadernos de Psicología del Deporte, 16(1), 211-222. https://doi.org/10.1037/ t56128-000

Rumbold, J., Fletcher, D. y Daniels, K. (2012). A systematic review of stress management interventions with sport performers.
Sport, Exercise, and Performance Psychology, 1(3), 173-193. https://doi.org/70.7037/a0026628

Rumbold, J., Fletcher, D. y Daniels, K. (2018). Using a mixed method audit to inform organizational stress management interventions in sport. Psychology of Sport and Exercise, 35(1), 27-38. https://doi.org/10.1016/j.psychsport.2017.10.010

Russell, M. T. y Karol, D. L. (2005). 16 PF-5. Manual. TEA Ediciones. Sherrington, C.S. (1961). Integrative action of the nervous system. Yale University Press.

Tabatabai, A. y Badami, R. (2019). Effects of Systematic Desensitization on state anxiety, fear of depth and interest to continue to sport participation in female swimming beginners. Journal of Clinical Psychology, 10(1), 59-68. https://doi.org/10.22075/ jcp.2019.13658.1339

Tabernero, B. y Márquez, S. (1994). Interrelación y cambios temporales en los componentes de la ansiedad estado competitiva. Revista de Psicología del Deporte, 6(1), 53-69.

Thomas, O., Mellalieu, S. D. y Hanton, S. (2009). Stress management in applied sport psychology. En S. D. Mellalieu y S. Hanton (Eds.), Advances in Applied Sport Psychology. A review (pp. 124-161). Routledge.

Vallejo-Slocker, L. y Vallejo, M. A. (2016). Sobre la desensibilización sistemática. Una técnica superada o renombrada. Acción Psicológica, 13(2), 157-168. https://doi.org/10.5944/ap.13.2.16539

Wolpe, J. (1958). Psychotherapy by reciprocal inhibition. Stanford University Press.

Wolpe, J. (1973). The practice of behavior therapy. Pergamon.

World Medical Association. (2013). World Medical Association declaration of Helsinki. Ethical principles for medical research involving human subjects. Journal of the American Medical Asociation, 310(20), 2191-2194. https://doi.org/10.1001/ jama.2013.281053

Zemore, R. (1975). Systematic desensitization as a method of teaching a general anxiety-reducing skill. Journal of Consulting and Clinical Psychology, 43(2), 157-161. https://doi.org/10.1037/ h0076534 